\title{
Opposite Skew left braces and applications
}

\author{
Alan Koch ${ }^{\mathrm{a}, *}$, Paul J. Truman ${ }^{\mathrm{b}}$ \\ ${ }^{a}$ Department of Mathematics, Agnes Scott College, 141 E. College Ave., Decatur, GA 30030 USA \\ ${ }^{b}$ School of Computing and Mathematics, Keele University, Staffordshire, ST5 5BG, UK
}

\begin{abstract}
Given a skew left brace $\mathfrak{B}$, we introduce the notion of an "opposite" skew left brace $\mathfrak{B}^{\prime}$, which is closely related to the concept of the opposite of a group, and provide several applications. Skew left braces are closely linked with both solutions to the Yang-Baxter Equation and Hopf-Galois structures on Galois field extensions. We show that the set-theoretic solution to the YBE given by $\mathfrak{B}^{\prime}$ is the inverse to the solution given by $\mathfrak{B}$. Every Hopf-Galois structure on a Galois field extension $L / K$ gives rise to a skew left brace $\mathfrak{B}$; if the underlying Hopf algebra is not commutative, then one can construct an additional, "commuting" Hopf-Galois structure (see [10], which relates the Hopf-Galois module structures of each); the corresponding skew left brace to this second structure is precisely $\mathfrak{B}^{\prime}$. We show how left ideals (and a newly introduced family of quasi-ideals) of $\mathfrak{B}^{\prime}$ allow us to identify the intermediate fields of $L / K$ which occur as fixed fields of sub-Hopf algebras under this correspondence. Finally, we use the opposite to connect the inverse solution to the YBE and the structure of the Hopf algebra $H$ acting on $L / K$; this allows us to identify the group-like elements of $H$.
\end{abstract}

Keywords: Skew left braces, Hopf-Galois structure, Yang-Baxter equation

2010 MSC: 16T25, 20N99, 16T05

\section{Introduction}

Skew left braces were developed by Guarnieri and Vendramin in [1] to construct non-degenerate, not necessarily involutive set-theoretic solutions to the Yang-Baxter equation. They were developed as a generalization to the concept of braces defined by Rump in [2] to find involutive solutions to the YBE. As first pointed out in [3, Remark 2.6] and developed in the appendix by Byott and Vendramin in [4], finite skew left braces-hereafter, "braces" for brevity-always arise from HopfGalois structures on Galois field extensions. In [3, Remark 2.6], the author writes "We hope that this connection between these two theories would be fruitful in the future", a hope which has been fulfilled: for example, in [5] Childs defines the notion of "circle-stable subgroups" of a brace and shows that such subgroups correspond to sub-Hopf algebras of the Hopf algebra giving the corresponding Hopf-Galois structure.

In this work (see Section 3), we introduce the rather simple notion of the opposite of a skew left brace. Our construction simply reverses the order in one of the two binary operations which deter-

\footnotetext{
${ }^{*}$ Corresponding author

Email addresses: akoch@agnesscott.edu (Alan Koch), P.J.Truman@Keele.ac.uk (Paul J. Truman)
} 
mine the brace. Our motivation comes from an existing pairing of non-commutative Hopf-Galois structures. We illustrate the usefulness of the opposite construction through a few applications.

As mentioned above, skew left braces provide set-theoretic solutions to the Yang-Baxter equation which are non-degenerate. Given a set $B$, a solution is a function $R: B \times B \rightarrow B \times B$ satisfying certain properties-see Section 2.2 for details. Each brace $\mathfrak{B}$ gives rise to such a solution $R_{\mathfrak{B}}$ : the non-degeneracy of $R_{\mathfrak{B}}$ implies that it has an inverse; in Section 4 we show how the opposite brace allows for an easy construction of $R_{\mathfrak{B}}^{-1}$.

Suppose $L / K$ is a finite Galois extension. Then Hopf-Galois structures on $L / K$ correspond with choices of certain groups $N$ of permutations of the elements of $\operatorname{Gal}(L / K)$, which in turn give rise to braces $\mathfrak{B}(N)$. Unlike classical Galois theory, a Hopf-Galois structure will give some, but not necessarily all, intermediate fields of $L / K$, only the ones which correspond to sub-Hopf algebras. It is natural to ask which intermediate fields arise, which [5] answers by constructing a new substructure of a brace. In Section 5 we use the opposite and relate these intermediate fields with the known brace substructure of ideals (and the closely related, new concept of quasi-ideals) of the opposite brace. Ideals allow us not only to find these intermediate fields $K \leq F \leq L$, but also single out, for example, which allow $L$ to be decomposed into two Galois extensions $L / F$ and $F / K$ which are also Hopf-Galois in a manner canonically related to the original Hopf-Galois structure.

One can also use the constructed solution to the YBE to understand some of the structure of the Hopf algebra which provides the corresponding Hopf-Galois structure. By using both connections to skew left braces, we are able to determine the group-like elements of the Hopf algebra by examining the second component of the solution to the YBE.

It is possible that a brace be equal to its own opposite, but it is easy to see that this happens if and only if a certain commutativity condition is satisfied. However, it is also possible to have a brace be isomorphic to its opposite, forming what we call, with abuse of terminology, a self-opposite brace. Knowing if a brace is self-opposite has important consequences when determining the intermediate fields in a Hopf-Galois extension which arise through the Hopf-Galois correspondence. Thus, in Section 6 we consider the self-opposite question. At this point, there seems to be no simple criterion to determine whether a brace is self-opposite.

\section{2. (Skew Left) Braces, the Yang-Baxter Equation, and Hopf-Galois Structures}

In this section we provide the background necessary for the rest of the paper.

\subsection{Braces}

We begin, of course, with the definition of a skew left brace. At this point, there does not seem to be standard notation for skew left braces; we set ours based on [1].

Definition 2.1. A skew left brace $\mathfrak{B}$ is a triple $(B, \cdot, \circ)$ consisting of a set and two binary operations, where $(B, \cdot)$ and $(B, \circ)$ are both groups and the following relation holds for all $x, y, z \in B$ :

$$
x \circ(y z)=(x \circ y) \cdot x^{-1} \cdot(x \circ z),
$$

where the symbol $x^{-1}$ refers to the inverse to $x \in(B, \cdot)$. We call the relation above the brace relation.

As one would expect, a brace homomorphism is a map preserving both the dot and circle operations, and an bijective homomorphism is a brace isomorphism. 
As stated in the introdution, for brevity we will refer to a skew left brace simply as a brace, however the reader should be aware that "(left) brace" is used by many to refer to the case where $(B, \cdot)$ is abelian as in [2].

Going forward, we will adopt the following notational conventions for $\mathfrak{B}=(B, \cdot, \circ)$, the first (mentioned above) included for completeness:

- For $x \in B$, the inverse to $x$ in $(B, \cdot)$ will be denoted $x^{-1}$.

- For $x \in B$, the inverse to $x$ in $(B, \circ)$ will be denoted $\bar{x}$.

- For $x, y \in B$ we will write $x y$ for $x \cdot y$ when no confusion can arise.

- The identity in both $(B, \cdot)$ and $(B, \circ)$ will be denoted $1_{B}$. Note that the symbol $1_{B}$ is not ambiguous: if $x \cdot 1_{B}=x$ for all $x \in B$ then

$$
x \circ 1_{B}=x \circ\left(1_{B} \cdot 1_{B}\right)=\left(x \circ 1_{B}\right) x^{-1}\left(x \circ 1_{B}\right),
$$

from which it follows from left cancellation that $x^{-1}\left(x \circ 1_{B}\right)=1_{B}$, i.e., $x=x \circ 1_{B}$.

Here are some examples which will be used throughout this paper.

Example 2.2. Let $(B, \cdot)$ be any finite group. Then $\mathfrak{B}=(B, \cdot, \cdot)$ is readily seen to be a brace. We call this the trivial brace on $B$.

Example 2.3. Let $(B, \cdot)$ be any finite group, and define $x \circ y=y \cdot x$ for all $x, y \in B$. Then $\mathfrak{B}=(B, \cdot, \circ)$ is also a brace. We call this the almost trivial brace on $B$.

Example 2.4. Let

$$
B=\left\langle\eta, \pi: \eta^{4}=\pi^{2}=\eta \pi \eta \pi=1\right\rangle .
$$

Then $B \cong D_{4}$, the dihedral group of order 8 . Define a binary operation $\circ$ on $B$ as follows:

$$
\eta^{i} \pi^{j} \circ \eta^{k} \pi^{\ell}=\eta^{2 j \ell}\left(\eta^{k} \pi^{\ell}\right)\left(\eta^{i} \pi^{j}\right)=\eta^{k+(-1)^{\ell} i+2 j \ell} \pi^{j+\ell} .
$$

Note that $\eta^{2 j \ell}$ is in the center of $(B, \cdot)$. This operation is associative: let $x_{j}=\eta^{i} \pi^{j}, x_{\ell}=\eta^{k} \pi^{\ell}$, and $x_{n}=\eta^{m} \pi^{n}$ for some choices $i, k, m$. Observe that, e.g., $x_{j} x_{\ell}=y_{j+\ell}$ for some $y_{j+\ell}=\eta^{r} \pi^{j+\ell}$. Then

$$
x_{j} \circ\left(x_{\ell} \circ x_{n}\right)=x_{j} \circ\left(\eta^{2 \ell n} x_{n} x_{\ell}\right)=\eta^{2 j(\ell+n)} \eta^{2 \ell n} x_{n} x_{\ell} x_{j}=\eta^{2 j \ell+2 j n+2 \ell n} x_{n} x_{\ell} x_{j}
$$

and similarly

$$
\left(x_{j} \circ x_{\ell}\right) \circ x_{n}=\eta^{2 j \ell} x_{\ell} x_{j} \circ x_{n}=\eta^{2 n(j+\ell)} \eta^{2 j \ell} x_{n} x_{\ell} x_{j}=\eta^{2 j \ell+2 j n+2 \ell n} x_{n} x_{\ell} x_{j} .
$$

Additionally, $\eta^{i} \pi^{j} \circ 1_{B}=\eta^{i} \pi^{j}$ so $1_{B}$ is the identity, and

$$
\eta^{i} \circ \eta^{-i}=1_{B}, \eta^{i} \pi \circ \eta^{i+2} \pi=1_{B}
$$

shows $\overline{\eta^{i}}=\eta^{-i}$ and $\overline{\eta^{i} \pi}=\eta^{i+2} \pi$ and hence $(B, \circ)$ is a group. The identities $\pi \circ \eta=\eta^{-1} \circ \pi$ and $\pi \circ \pi=\eta \circ \eta$ can be easily established, and since

$$
\eta^{\circ k}:=\underbrace{\eta \circ \eta \cdots \circ \eta}_{k \text { times }}=\eta^{k}
$$


we see $\eta \in(B, \circ)$ has order 4 , hence $(B, \circ) \cong Q_{8}$.

Finally, we claim that $(B, \cdot \circ)$ satisfies the brace relation. Writing $x_{j}, x_{\ell}$, and $x_{n}$ as above we get

$$
\begin{aligned}
x_{j} \circ\left(x_{\ell} x_{n}\right) & =\eta^{2 j(\ell+n)} x_{\ell} x_{n} x_{j} \\
& =\eta^{2 j \ell} x_{\ell} \eta^{2 j n} x_{n} x_{j} \\
& =\eta^{2 j \ell} x_{\ell}\left(x_{j} x_{j}^{-1}\right) \eta^{2 j n} x_{n} x_{j} \\
& =\left(x_{j} \circ x_{\ell}\right) x_{j}^{-1}\left(x_{j} \circ x_{n}\right),
\end{aligned}
$$

and hence $(B, \cdot, \circ)$ is a brace.

\subsection{The Yang-Baxter Equation}

As mentioned previously, skew left braces were originally constructed to provide set-theoretic solutions to the Yang-Baxter Equation. We now review this concept.

Definition 2.5. A set-theoretic solution to the Yang-Baxter equation is a set $B$ together with a function $R: B \times B \rightarrow B \times B$ such that

$$
R_{12} R_{23} R_{12}(x, y, z)=R_{23} R_{12} R_{23}(x, y, z)
$$

for all $x, y, z \in B$, where $R_{12}=R \times 1_{B}$ and $R_{23}=1_{B} \times R$.

Furthermore, we say $R$ is involutive if $R(R(x, y))=(x, y)$ for all $x, y \in B$; and if we write $R(x, y)=\left(f_{x}(y), f_{y}(x)\right)$ for some functions $f_{x}, f_{y}: B \rightarrow B$ we say $R$ is non-degenerate if $f_{x}$ and $f_{y}$ are both bijections.

Notice above that we will often refer to $R$ as the solution, leaving $B$ implicit.

Example 2.6. Let $B$ be any finite group, written multiplicatively. Then $R(x, y)=\left(y, y^{-1} x y\right)$ is a non-degenerate solution to the YBE. It is involutive if and only if $B$ is abelian.

Example 2.7. In a manner similar to the above, let $B$ be any finite group, written multiplicatively. Then $R(x, y)=\left(x^{-1} y x, x\right)$ is a non-degenerate solution to the YBE. It is also involutive if and only if $B$ is abelian.

Example 2.8. Let $B$ be the set $B=\left\{\eta^{i} \pi^{j}: 0 \leq i \leq 3,0 \leq \pi \leq 1\right\}$. Then

$$
R\left(\eta^{i} \pi^{j}, \eta^{k} \pi^{\ell}\right)=\left(\eta^{(-1)^{j} k+2 i \ell+2 j \ell} \pi^{\ell}, \eta^{i+2 j \ell} \pi^{j}\right)
$$

provides a non-degenerate solution to the YBE, where the exponent on $\eta$ is interpreted $\bmod 4$ and the exponent on $\pi$ is interpreted $\bmod 2$. We leave the details to the reader for now, although it will follow from the paragraph to follow that $R$ must satisfy with YBE.

The connection between solutions to the YBE and braces are as follows. Suppose $\mathfrak{B}:=(B, \cdot, \circ)$ is a brace. Let $R_{\mathfrak{B}}: B \times B \rightarrow B \times B$ be given by

$$
R_{\mathfrak{B}}(x, y)=\left(x^{-1}(x \circ y), \overline{x^{-1}(x \circ y)} \circ x \circ y\right) .
$$

By [1, Theorem 1], $R_{\mathfrak{B}}$ provides a non-degenerate, set-theoretic solution to the YBE, involutive if and only if $(B, \cdot)$ is abelian. In fact, Examples 2.6, 2.7, 2.8 were constructed from the braces given in Examples 2.2, 2.3, and 2.4 respectively. 


\subsection{Hopf-Galois Structures}

We start by recalling the definition of a Hopf-Galois extension-more details can be found, e.g., in $[6, \S 2]$.

Definition 2.9. Let $L / K$ be a field extension. Suppose there exists a $K$-Hopf algebra $H$, with comultiplication and counit maps $\Delta$ and $\varepsilon$ respectively, which acts on $L$ such that

1. $h \cdot(s t)=$ mult $\Delta(h)(s \otimes t), h \in H, s, t \in L$,

2. $h(1)=\varepsilon(h) 1, h \in H$,

3. The $K$-module homomorphism $L \otimes_{K} H \rightarrow \operatorname{End}_{K}(L)$ given by $(s \otimes h)(t)=s h(t), h \in H, s, t \in$ $L$ is an isomorphism.

Then $H$ is said to provide a Hopf-Galois structure on $L / K$, and we say $L / K$ is Hopf-Galois with respect to $H$, or $H$-Galois.

If $H$ gives a Hopf-Galois structure on $L / K$ then

$$
L^{H}:=\{s \in L: h(s)=\varepsilon(h) s \text { for all } h \in H\}=K,
$$

and we think of $K$ as the "fixed field" under this action. If $H_{0}$ is a sub-Hopf algebra of $H$, then $L^{H_{0}}$, defined analogously, is an intermediate field in the extension $L / K$. While the usual Galois correspondence provides a bijection between subgroups and intermediate fields, the correspondence between sub-Hopf algebras and intermediate fields need not be onto (though it is certainly injective).

In the groundbreaking paper [7], Greither and Pareigis showed that Hopf-Galois structures on any separable field extension $L / K$ could be found using only group theory; we shall outline their results in the case where $L / K$ is Galois. Let $G=\operatorname{Gal}(L / K)$, and let $\operatorname{Perm}(G)$ denote the group of permutations of $G$. A subgroup $N \leq \operatorname{Perm}(G)$ is called regular if for all $g, h \in G$ there exists a unique $\eta \in N$ such that $\eta[g]=h$. Note that $N$ must have the same order as $G$. Furthermore, we shall say $N$ is $G$-stable if ${ }^{g} \eta \in N$ for all $g \in G, \eta \in N$, where ${ }^{g} \eta \in \operatorname{Perm}(G)$ is given by

$$
{ }^{g} \eta[h]=\lambda(g) \eta \lambda\left(g^{-1}\right)[h], h \in G
$$

and $\lambda(k) \in \operatorname{Perm}(G)$ is left multiplication by $k \in G$.

Given a regular, $G$-stable $N \leq \operatorname{Perm}(G)$, let $H_{N}$ be the invariant $\operatorname{ring} H_{N}=L[N]^{G}$, where $G$ acts on $N$ as above and on $L$ through Galois action. Then $H_{N}$ is a $K$-Hopf algebra which acts on $\ell \in L$ via

$$
\left(\sum_{\eta \in N} a_{\eta} \eta\right) \cdot \ell=\sum_{\eta \in N} a_{\eta} \eta^{-1}\left[1_{G}\right](\ell), \sum_{\eta \in N} a_{\eta} \eta \in H_{N} \subset L[N]^{G} .
$$

The association $N \mapsto H_{N}$ for $N \leq \operatorname{Perm}(G)$ is a bijection between regular, $G$-stable subgroups and Hopf Galois structures on $L / K$.

Example 2.10. Let $N=\rho(G)=\{\rho(g): g \in G\}$, where $\rho(g)[h]=h g^{-1}$ is right regular representation. For $h, k \in G$ then $\rho(g)[h]=k$ if and only if $g=h k^{-1}$, hence $\rho(G)$ is regular. Since the images of the left and right regular representations commute, $\rho(G)$ is $G$-stable. In fact, $\lambda(g)$ acts trivially on $\rho(h)$ for all $g, h \in G$, so $H_{\rho(G)} \cong K[G]$. Using the formula given above we see that the action of $H_{\rho(G)}$ on $L$ corresponds to the usual action of $K[G]$, and so we recover the classical Galois structure on $L / K$. 
Example 2.11. Let $N=\lambda(G)=\{\lambda(g): g \in G\}, \lambda(g)$ as above. Then $\lambda(G)$ is regular, and since ${ }^{g} \lambda(h)=\lambda\left(g h g^{-1}\right) \in \lambda(G)$ we see that $\lambda(G)$ is a $G$-stable subgroup of Perm $(G)$. The structure given by $H_{\lambda(G)}$ is called the canonical nonclassical Hopf-Galois structure in [8].

Example 2.12. Suppose $G=\left\langle s, t: s^{4}=t^{4}=1, s^{2}=t^{2}\right.$, stst $\left.t^{-1}=1\right\rangle \cong Q_{8}$. Let $\eta=\rho(s), \pi=$ $\lambda(s) \rho(t) \in \operatorname{Perm}(G)$, and let $N=\langle\eta, \pi\rangle$. Then $N \leq \operatorname{Perm}(G)$ is regular, $G$-stable, and $N \cong D_{4}$, the dihedral group of order 4: see [9, Lemma 2.5] for details. Note that this is one of many regular, $G$-stable subgroups of $\operatorname{Perm}(G)$, as found in loc. cit.

\subsection{Connecting Braces to Hopf-Galois Structures}

As mentioned in the introduction, Bachiller points out a connection between Hopf-Galois structures and braces. We shall describe this connection using an equivalent, but different, formulation of the correspondence.

Let $*_{G}$ denote the group operation on some finite group $G$, and suppose $(N, \cdot) \leq \operatorname{Perm}(G)$ is regular and $G$-stable. Then there is a map $a: N \rightarrow G$ given by

$$
a(\eta)=\eta\left[1_{G}\right] .
$$

By the regularity of $N, a$ is a bijection. We define a binary operation $\circ$ on $N$ by

$$
\eta \circ \pi=a^{-1}\left(a(\eta) *_{G} a(\pi)\right), \eta, \pi \in N .
$$

Then $(N, \circ) \cong\left(G, *_{G}\right)$, and $(N, \cdot, \circ)$ is a brace-note that $G$-stability is used in verifying that the brace relation holds. We shall denote this brace by $\mathfrak{B}(N)$, which we understand depends implictly on $G$. As every Hopf-Galois structure on a Galois extension with group $G$ corresponds to a regular, $G$-stable $N$ we get can construct a brace for every such structure.

Example 2.13. Let $N=\rho(G)=\{\rho(g): g \in G\}$, where $\rho(g)[h]=h g^{-1}$ is right regular representation. Then $a: \rho(G) \rightarrow G$ is the "inverse" map $a(\rho(g))=g^{-1}$, and the corresponding brace has circle operation

$$
\rho(g) \circ \rho(h)=a^{-1}(a(\rho(g)) a(\rho(h)))=a^{-1}\left(g^{-1} h^{-1}\right)=\rho\left(\left(g^{-1} h^{-1}\right)^{-1}\right)=\rho(h g)=\rho(h) \rho(g)
$$

giving the almost trivial brace constructed in Example 2.3.

Example 2.14. Let $N=\lambda(G)$, so $a: N \rightarrow G$ is simply $a(\lambda(g))=g$. Then

$$
\lambda(g) \circ \lambda(h)=a^{-1}(a(\lambda(g)) a(\lambda(h)))=a^{-1}(g h)=\lambda(g h)=\lambda(g) \lambda(h)
$$

giving the trivial brace from Example 2.2

Example 2.15. Let $G, N$ be as in Example 2.12. Then $a: N \rightarrow G$ is given by

$$
a\left(\eta^{i}\right)=\eta^{i}\left[1_{G}\right]=s^{-i}, a\left(\eta^{i} \pi\right)=\eta^{i} \pi\left[1_{G}\right]=\eta^{i}\left[s t^{-1}\right]=s t^{-1} s^{-i}=s^{i+1} t^{-1} .
$$

It is easiest to work out the circle operation in cases, depending on the powers of $\pi$. We have

$$
\begin{aligned}
\eta^{i} \circ \eta^{k} & =a^{-1}\left(s^{-i-k}\right)=\eta^{i+k} \\
\eta^{i} \circ \eta^{k} \pi & =a^{-1}\left(s^{-i+k+1} t^{-1}\right)=\eta^{k-i} \pi \\
\eta^{i} \pi \circ \eta^{k} & =a^{-1}\left(s^{i+1} t^{-1} s^{-k}\right)=a^{-1}\left(s^{i+k+1} t^{-1}\right)=\eta^{i+k} \pi \\
\eta^{i} \pi \circ \eta^{k} \pi & =a^{-1}\left(s^{i+1} t^{-1} s^{k+1} t^{-1}\right)=a^{-1}\left(s^{i-k} t^{-2}\right)=a^{-1}\left(s^{i-k+2}\right)=\eta^{k-i-2} .
\end{aligned}
$$


Generally,

$$
\eta^{i} \pi^{j} \circ \eta^{k} \pi^{\ell}=\eta^{k+(-1)^{\ell} i+2 j \ell} \pi^{j+\ell},
$$

which agrees with the brace constructed in Example 2.4.

Conversely, suppose $\mathfrak{B}=(B, \cdot, \circ)$ is a brace. Then $(B, \circ)$ is a group. For each $x \in B$ define $\eta_{x} \in \operatorname{Perm}(B, \circ)$ by

$$
\eta_{x}[y]=x \cdot y, y \in B .
$$

Then $\eta_{x}[y]=z$ if and only if $x=z \cdot y^{-1}$, so $N=\left\{\eta_{x}: x \in B\right\}$ is a regular subgroup of $\operatorname{Perm}(B, \circ)$. Furthermore, $N$ is $(B, \circ)$-stable: for $x, y \in B$ we have, since $\lambda(y) \in \operatorname{Perm}(B, \circ)$ is left multiplication in the circle group,

$$
\begin{aligned}
{ }^{y} \eta_{x}[z] & =\lambda(y) \eta_{x} \lambda(\bar{y})[z] \\
& =\lambda(y) \eta_{x}[\bar{y} \circ z] \\
& =\lambda(y)[x \cdot(\bar{y} \circ z)] \\
& =y \circ(x \cdot(\bar{y} \circ z)) \\
& =(y \circ x) y^{-1}(y \circ \bar{y} \circ z) \\
& =(y \circ x) y^{-1} \cdot z \\
& =\eta_{(y \circ x) y^{-1}[z],}
\end{aligned}
$$

so ${ }^{y} \eta_{x}=\eta_{(y \circ x) y^{-1}} \in N$. Thus, $N$ is a regular, $(B, \circ)$-stable subgroup of $\operatorname{Perm}(B, \circ)$, hence $N$ provides a Hopf-Galois structure on any Galois extension $L / K$ with Galois group isomorphic to $(B, \circ)$.

Example 2.16. Let $G=\langle s, t\rangle \cong Q_{8}$ as in Example 2.12. Let $\eta_{t}=\rho(t), \pi_{t}=\lambda(t) \rho(s)$, and let $N_{t}=\left\langle\eta_{t}, \pi_{t}\right\rangle$. Then, by [9, Lemma 2.5], $N_{t} \leq \operatorname{Perm}(G)$ is regular, $G$-stable, isomorphic to $D_{4}$, but different from the one considered in Example 2.12. Proceeding in a manner similar to 2.15 one can show

$$
\eta_{t}^{i} \pi_{t}^{j} \circ \eta_{t}^{k} \pi_{t}^{\ell}=\eta_{t}^{k+(-1)^{\ell} i+2 j \ell} \pi_{t}^{j+\ell},
$$

and thus we see that different Hopf-Galois structures can give the same brace.

\section{The Opposite Brace}

In this section, we shall define the opposite brace and describe some of its properties.

Proposition 3.1. Let $\mathfrak{B}=(B, \cdot, \circ)$ be a brace, and for each $x, y \in B$ define $x \cdot^{\prime} y=y x$. Then $\mathfrak{B}^{\prime}:=\left(B,{ }^{\prime}, \circ\right)$ is a brace.

Proof. Clearly, $(B, \circ)$ is a group, and since $\left(B,,^{\prime}\right)$ is the opposite group of $(B, \cdot)$ it is a group as well, sharing the same identity and inverses. It remains to show the brace relation. For $x, y, z \in B$ we have, using the brace relation on $\mathfrak{B}$,

$$
\begin{aligned}
x \circ\left(y \bullet^{\prime} z\right) & =x \circ(z y) \\
& =(x \circ z) x^{-1}(x \circ y) \\
& =(x \circ z) \cdot\left((x \circ y) \cdot^{\prime} x^{-1}\right) \\
& =\left((x \circ y) \cdot^{\prime} x^{-1}\right) \cdot^{\prime}(x \circ z) \\
& =(x \circ y) \cdot^{\prime} x^{-1} \bullet^{\prime}(x \circ z),
\end{aligned}
$$


and hence $\mathfrak{B}^{\prime}$ is a brace.

Definition 3.2. For $\mathfrak{B}=(B, \cdot, \circ)$ a brace, the brace $\mathfrak{B}^{\prime}$ constructed above is called the opposite brace to $\mathfrak{B}$.

We list the following properties for future reference. Their proofs are trivial and omitted.

Lemma 3.3. Let $\mathfrak{B}=(B, \cdot, \circ)$ be a brace. Then

1. $\left(\mathfrak{B}^{\prime}\right)^{\prime}=\mathfrak{B}$.

2. If $(B, \cdot)$ is abelian, then $\mathfrak{B}^{\prime}=\mathfrak{B}$.

3. If $\mathfrak{C}$ is a brace, and $f: \mathfrak{B} \rightarrow \mathfrak{C}$ is a brace homomorphism, then $f$ is also a brace homomorphism $\mathfrak{B}^{\prime} \rightarrow \mathfrak{C}^{\prime}$.

Opposite braces arise arise from an existing construction in Hopf-Galois theory, which we term the opposite Hopf Galois structure, which we shall now describe. Let $G$ be a group, and let $N \leq$ $\operatorname{Perm}(G)$ be regular and $G$-stable. Define

$$
N^{\prime}=\operatorname{Cent}_{\operatorname{Perm}(G)}(N)=\left\{\eta^{\prime} \in \operatorname{Perm}(G): \eta \eta^{\prime}=\eta^{\prime} \eta \text { for all } \eta \in N\right\} .
$$

Then, by [7, Lemmas 2.4.1, 2.4.2], $N^{\prime}$ is a regular, $G$-stable subgroup of $\operatorname{Perm}(G)$. In fact, for $\eta \in N$, define $\phi_{\eta} \in \operatorname{Perm}(G)$ by $\phi_{\eta}[g]=\mu_{g}\left[\eta\left[1_{G}\right]\right]$, where $\mu_{g}$ is the element of $N$ such that $\mu_{g}(1)=g$ (such a $\mu_{g}$ exists, and is unique, by regularity). One can show that $\phi_{\eta} \phi_{\pi}=\phi_{\pi \eta}$ for $\eta, \pi \in N$, and that $N^{\prime}$ naturally identifies with the opposite group $N^{\text {opp }}$ of $N$. The relationship between $N$ and $N^{\prime}$ has been explored in the area of Hopf-Galois module theory, producing some interesting results [10].

Let us compute the brace corresponding to $N^{\prime}$. Let $a: N \rightarrow G$ and $a^{\prime}: N^{\prime} \rightarrow G$ be the bijections obtained by evaluation at $1_{G}$ as before. Then

$$
a^{\prime}\left(\phi_{\eta}\right)=\phi_{\eta}\left[1_{G}\right]=\mu_{1_{G}}\left[\eta\left[1_{G}\right]\right]=1_{N}\left[\eta\left[1_{G}\right]\right]=\eta\left[1_{G}\right]=a(\eta),
$$

hence $\mathfrak{B}\left(N^{\prime}\right)=\left(N^{\prime}, \cdot, o^{\prime}\right)$ with

$$
\begin{aligned}
\phi_{\eta} \circ^{\prime} \phi_{\pi} & =\left(a^{\prime}\right)^{-1}\left(a^{\prime}\left(\phi_{\eta}\right) a^{\prime}\left(\phi_{\pi}\right)\right) \\
& =\left(a^{\prime}\right)^{-1}(a(\eta) a(\pi)) \\
& =\left(a^{\prime}\right)^{-1} a a^{-1}(a(\eta) a(\pi)) \\
& =\left(a^{\prime}\right)^{-1} a(\eta \circ \pi) \\
& =\phi_{\eta \circ \pi} .
\end{aligned}
$$

Define $f: \mathfrak{B}\left(N^{\prime}\right) \rightarrow(\mathfrak{B}(N))^{\prime}$ by $f\left(\phi_{\eta}\right)=\eta$ for all $\eta \in N$. Then

$$
f\left(\phi_{\eta} \circ \phi_{\pi}\right)=f\left(\phi_{\eta \circ \pi}\right)=\eta \circ \pi=f\left(\phi_{\eta}\right) \circ f\left(\phi_{\pi}\right)
$$

and

$$
f\left(\phi_{\eta} \phi_{\pi}\right)=f\left(\phi_{\pi \eta}\right)=\pi \eta=\eta \cdot^{\prime} \pi=f(\eta) \cdot^{\prime} f(\pi)
$$

for all $\eta, \pi \in N$. Thus:

Proposition 3.4. With the notation as above, $\mathfrak{B}\left(N^{\prime}\right) \cong(\mathfrak{B}(N))^{\prime}$. 
Example 3.5. Let $G=\left\langle s, t: s^{4}=t^{4}=1, s^{2}=t^{2}\right.$, stst $\left.t^{-1}\right\rangle \cong Q_{8}$ as in Example 2.12. In [9, Lemma $2.5]$ one finds six different regular, $G$-stable subgroups which are isomorphic to $D_{4}$, namely

$$
\begin{array}{lll}
N_{s, \rho}=\langle\rho(s), \lambda(s) \rho(t)\rangle & N_{t, \rho}=\langle\rho(t), \lambda(t) \rho(s)\rangle & N_{s t, \rho}=\langle\rho(s t), \lambda(s t) \rho(t)\rangle \\
N_{s, \lambda}=\langle\lambda(s), \lambda(t) \rho(s)\rangle & N_{t, \lambda}=\langle\lambda(t), \lambda(s) \rho(t)\rangle & N_{s t, \lambda}=\langle\lambda(s t), \lambda(t) \rho(s t)\rangle .
\end{array}
$$

Note that the first two correspond to Examples 2.12 and 2.16 respectively. We have seen that $\mathfrak{B}\left(N_{s, \rho}\right) \cong \mathfrak{B}\left(N_{t, \rho}\right)$, and it is easy to see that they are isomorphic to $\mathfrak{B}\left(N_{s t, \rho}\right)$ as well. One can quickly verify that the elements of $N_{x, \rho}$ and $N_{x, \lambda}$ commute for each $x \in\{s, t, s t\}$, hence the three subgroups in the second row all correspond (up to isomorphism) the same brace, namely $\mathfrak{B}\left(N_{s, \rho}\right)^{\prime}$.

\section{The Inverse Solution to the Yang-Baxter Equation}

Earlier, we saw how a brace $\mathfrak{B}:=(B, \cdot, \circ)$ provides us with a set-theoretic solution $R_{\mathfrak{B}}$ to the YBE: one which is always non-degenerate, and one which is involutive (that is, self-inverse) if and only if $(B, \cdot)$ is abelian. It is natural to wonder what the inverse to $R_{\mathfrak{B}}$ is when $(B, \cdot)$ is not abelian. Since $\mathfrak{B}=\mathfrak{B}^{\prime}$ if and only if $(B, \cdot)$ is abelian, perhaps the opposite brace can help us determine the inverse. In fact:

Theorem 4.1. Let $\mathfrak{B}$ be a brace with corresponding solution to the Yang-Baxter equation $R_{\mathfrak{B}}$. Then $R_{\mathfrak{B}^{\prime}}$ is a two-sided inverse to $R_{\mathfrak{B}}$, that is, $R_{\mathfrak{B}^{\prime}} R_{\mathfrak{B}}(x, y)=R_{\mathfrak{B}} R_{\mathfrak{B}^{\prime}}(x, y)=(x, y)$ for all $x, y \in B$.

Proof. By interchanging a brace with its opposite, it suffices to show that $R_{\mathfrak{B}^{\prime}} R_{\mathfrak{B}}(x, y)=(x, y)$ for all $x, y \in B$. Recall that both $\mathfrak{B}$ and $\mathfrak{B}^{\prime}$ have the same inverses, i.e., $x \cdot^{\prime} x^{-1}=x \circ \bar{x}=1_{B}$ where $x^{-1}, \bar{x}$ are the inverses in $\mathfrak{B}$.

Let $x, y \in B$. We have

$$
\begin{aligned}
R_{\mathfrak{B}}(x, y) & =\left(x^{-1} \cdot(x \circ y), \overline{x^{-1} \cdot(x \circ y)} \circ x \circ y\right) \\
R_{\mathfrak{B}^{\prime}}(x, y) & =\left(x^{-1} \cdot^{\prime}(x \circ y), \overline{x^{-1} \cdot(x \circ y)} \circ x \circ y\right)=\left((x \circ y) \cdot x^{-1}, \overline{(x \circ y) \cdot x^{-1}} \circ x \circ y\right)
\end{aligned}
$$

and so, suppressing the dot notation,

$$
R_{\mathfrak{B}^{\prime}} R_{\mathfrak{B}}(x, y)=R_{\mathfrak{B}^{\prime}}\left(x^{-1}(x \circ y), \overline{x^{-1}(x \circ y)} \circ x \circ y\right) .
$$

The first component of this composition is therefore

$$
\left(\left(x^{-1}(x \circ y)\right) \circ\left(\overline{x^{-1}(x \circ y)} \circ x \circ y\right)\right)\left(x^{-1}(x \circ y)\right)^{-1}=(x \circ y)(x \circ y)^{-1} x=x,
$$

while the second component, using the reduction above, is

$$
\bar{x} \circ x \circ y=y,
$$

as required.

Example 4.2. Return to the solution $R_{\mathfrak{B}}$ from Example 2.8, namely

$$
R\left(\eta^{i} \pi^{j}, \eta^{k} \pi^{\ell}\right)=\left(\eta^{(-1)^{j} k+2 i \ell+2 j \ell} \pi^{\ell}, \eta^{i-2 j \ell} \pi^{j}\right),
$$


which was obtained from the brace in Example 2.4. The reader can check that we have

$$
R_{\mathfrak{B}^{\prime}}\left(\eta^{i} \pi^{j}, \eta^{k} \pi^{\ell}\right)=\left(\eta^{k+2 j \ell} \pi^{\ell}, \eta^{(-1)^{\ell} i+2 j k+2 j \ell} \pi^{j}\right) .
$$

To verify that $R_{\mathfrak{B}^{\prime}}=R_{\mathfrak{B}}^{-1}$, we have

$$
\begin{aligned}
R_{\mathfrak{B}^{\prime}} R_{\mathfrak{B}}\left(\eta^{i} \pi^{j}\right) & =R_{\mathfrak{B}^{\prime}}\left(\eta^{(-1)^{j} k+2 i \ell+2 j \ell} \pi^{\ell}, \eta^{i-2 j \ell} \pi^{j}\right) \\
& =\left(\eta^{i+2 j \ell-2 j \ell} \pi^{j}, \eta^{(-1)^{j}\left[(-1)^{j} k+2 i \ell+2 j \ell\right]+2 \ell(i-2 j \ell)-2 j \ell} \pi^{\ell}\right) \\
& =\left(\eta^{i}, \eta^{k+(-1)^{j}[2 i \ell+2 j \ell]+2 i \ell-2 j \ell} \pi^{\ell}\right) \\
& =\left(\eta^{i} \pi^{j}, \eta^{k} \pi^{\ell}\right)
\end{aligned}
$$

since $\eta^{4}=1_{B}$. That $R_{\mathfrak{B}^{\prime}} R_{\mathfrak{B}}\left(\eta^{i} \pi^{j}\right)=\left(\eta^{i}, \pi^{j}\right)$ is similar.

The explicit inverse solution allows us to identify group-like elements in the corresponding Hopf algebra. Recall that $h \in H$ is group-like if $\Delta(h)=h \otimes h$ where $\Delta$ is the comultiplication in the Hopf algebra $H$.

Corollary 4.3. Let the Galois extension $L / K$ be $H$-Hopf Galois for some $K$-Hopf algebra $H_{N}$. Let $\mathfrak{B}=(B, \cdot, \circ)$ be the brace corresponding to this Hopf-Galois structure, and for $i=1,2$ let $\operatorname{pr}_{i}: B \times B \rightarrow B$ be the projection onto the $i^{\text {th }}$ factor. Then each $y \in B$ with $\operatorname{pr}_{2} R_{\mathfrak{B}}(x, y)=x$ for all $x$ naturally identifies with a group-like element of $H_{N}$, and vice-versa.

Proof. We claim that an element $h \in H_{N}=L[N]^{G}$ is group-like if and only if $h \in N$ and $G$ acts trivially upon it, that is, if and only if $h \in N \cap \rho(G)$. Indeed, $h \in H_{N}$ is group-like if and only if it is group-like when base changed to $L \otimes_{K} L[N]^{G} \cong L[N]$, and since the group-likes in $L[N]$ are the elements of the group $N$ it follows that $h$ is group-like if and only if $h \in N$, say $h=\eta \in N$. But $G$ acts trivially on $\eta$ if and only if $\lambda(g) \eta \lambda\left(g^{-1}\right)=\eta$ for all $g \in G$, i.e., $\eta \in \operatorname{Cent}_{\operatorname{Perm}(G)}(\lambda(G))=\rho(G)$.

Recall that $\mathfrak{B}$ induces a regular, $(B, \circ)$ stable subgroup of $\operatorname{Perm}(B, \circ): N=\left\{\eta_{y}: y \in B\right\} \leq$ $\operatorname{Perm}(B, \circ)$ where $\eta_{y}[z]=y \cdot z$, and ${ }^{x} \eta_{y}=\eta_{(x \circ y) x^{-1}}$. So $(B, \circ)$ acts trivially on $\eta_{y}$ if any only if $(x \circ y) x^{-1}=y$, i.e., $\operatorname{pr}_{1} R_{\mathfrak{B}^{\prime}}(x, y)=y$ for all $x \in B$. This can only happen if $\operatorname{pr}_{2} R_{\mathfrak{B}}(x, y)=x$ since $R_{\mathfrak{B}} R_{\mathfrak{B}^{\prime}}(x, y)=(x, y)$. Through the isomorphism $(B, \circ) \rightarrow G$ we obtain the grouplike in $H_{N}$.

Example 4.4. The trivial brace, corresponding to $N=\lambda(G)$, gives the solution $R(x, y)=$ $\left(y, y^{-1} x y\right)$. So $y$ is group-like if and only if $y^{-1} x y=x$ for all $x \in B$, i.e., $y \in Z(B, \cdot)$.

Example 4.5. The almost trivial brace, corresponding to $N=\rho(G)$ and the classical Galois structure, gives the solution $R(x, y)=\left(x^{-1} y x, x\right)$. Clearly, every $y$ is group-like.

Example 4.6. The brace considered in Example 2.4, corresponding to the Hopf-Galois structure in Example 2.12, gives the solution

$$
R\left(\eta^{i} \pi^{j}, \eta^{k} \pi^{\ell}\right)=\left(\eta^{(-1)^{j} k+2 i \ell+2 j \ell} \pi^{\ell}, \eta^{i+2 j \ell} \pi^{j}\right) .
$$

One can see that $\operatorname{pr}_{2} R\left(\eta^{i} \pi^{j}, \eta^{k} \pi^{\ell}\right)=\eta^{i} \pi^{j}$ for all $i, j$ if and only if $\ell$ is even, hence the group-likes correspond are elements of the form $\eta^{k}$. This makes sense since $\eta=\rho(s)$. 


\section{On the Hopf-Galois Correspondence}

Suppose $L / K$ is Galois with Galois group $G$. We have seen that any $N \leq \operatorname{Perm}(G)$ regular, $G$-stable gives rise to a Hopf-Galois structure on $L / K$, but the correspondence between sub-Hopf algebras and intermediate fields is not surjective. It is natural to ask: which intermediate fields arise as the fixed field of a sub-Hopf algebra? Since the correspondence from sub-Hopf algebras to intermediate fields is injective, this is equivalent to determining the sub-Hopf algebras of $H_{N}$.

Definition 5.1. Let $L / K$ be Hopf-Galois for some Hopf algebra $H$. We say that an intermediate field $K \subseteq F \subseteq L$ is realizable with respect to $H$ (or simply realizable for short) if $F=L^{H_{0}}$ for $H_{0}$ some sub-Hopf algebra of $H$.

In [5], Childs shows that realizable subfields are in one-to-one correspondence with what he calls "o-stable (or "circle-stable') subgroups" of the corresponding brace. For $\mathfrak{B}=\mathfrak{B}(N)=(B, \cdot, \circ)$, a subgroup $C \leq(B, \cdot)$ is said to be o-stable if $(x \circ y) x^{-1} \in C$ for $x \in B, y \in C$. A o-stable subgroup is closed under $\circ$ as well, hence is a sub-brace of $\mathfrak{B}$.

We will take a different approach to realizable subfields using the results of [11] and the concept of opposites. It is not hard to show that a o-stable subgroup, when viewed in the opposite brace, looks like the more familiar brace structure called an ideal, one which we generalize somewhat below by relaxing normality conditions.

Definition 5.2. Let $\mathfrak{B}=(B, \cdot, \circ)$ be a brace.

1. A quasi-ideal of $\mathfrak{B}$ is a subgroup $I \leq(B, \cdot)$ such that

$$
x^{-1}(x \circ y) \in I, x \in B, y \in I .
$$

2. A --quasi-ideal (--QI) of $\mathfrak{B}$ is a quasi ideal which is normal in $(B, \cdot)$.

3. A o-quasi-ideal (o-QI) of $\mathfrak{B}$ is a quasi ideal which is normal in $(B, \circ)$.

4. An ideal of $\mathfrak{B}$ is a subgroup of $(B, \cdot)$ which is both a $\cdot-\mathrm{QI}$ and a o-QI.

Note that a quasi-ideal $I$ is also a subgroup of $(B, \circ)$, hence is a sub-brace of $\mathfrak{B}$. To see this, note that for all $x, y, z \in B$ we have

$$
x^{-1}(x \circ y \circ z)=x^{-1}(x \circ y)(x \circ y)^{-1}(x \circ y \circ z),
$$

and if $y, z \in I$ then $x^{-1}(x \circ y) \in I$ and $(x \circ y)^{-1}(x \circ y \circ z) \in I$, hence their product is in $I$ and $I$ is closed under $\circ$. Additionally,

$$
1_{B}=x^{-1}(x \circ y \bar{y})=x^{-1}(x \circ y) x^{-1}(x \circ \bar{y},
$$

and since $1_{B} \in I$ and $x^{-1}(x \circ y) \in I$ we get that $x^{-1}(x \circ \bar{y}) \in I$, i.e., $\bar{y} \in I$.

By [1, Example 2.2], the kernel of a brace morphism has the structure of an ideal. Additionally, by [1, Lemma 2.3], if $I$ is an ideal of $\mathfrak{B}$ then both $I$ and $B / I$ are braces. Thus, ideals are essential to understanding the category of braces.

Now suppose $\mathfrak{B}=\mathfrak{B}(N)$ for $N$ a regular $G$-stable subgroup of $\operatorname{Perm}(G)$, where $G:=\operatorname{Gal}(L / K)$. Each of the substructures above gives us insight as to the intermediate fields in the $H_{N^{\prime}}$-Hopf Galois structure on $L / K$, where as above $N^{\prime}=\operatorname{Cent}_{\operatorname{Perm}(G)}(N)$ as before.

We begin with the simplest of the structures. 
Lemma 5.3. Quasi-ideals $I$ of $\mathfrak{B}:=\mathfrak{B}(N)$ correspond bijectively with intermediate fields $K \leq$ $L_{I} \leq L$ realizable with respect to $H_{N^{\prime}}$.

Proof. Let $I$ be a quasi-ideal of $\mathfrak{B}$. Since the underlying sets of $\mathfrak{B}$ and $\mathfrak{B}^{\prime}$ are the same, namely $N$, and $x^{-1}(x \circ y)=(x \circ y){ }^{\prime} x^{-1}$ for all $x, y \in N$ we get that $I$ is a o-stable subgroup of $\mathfrak{B}^{\prime}$. Through the isomorphism $(\mathfrak{B}(N))^{\prime} \rightarrow \mathfrak{B}\left(N^{\prime}\right)$ its image is a o-stable subgroup in $\mathfrak{B}\left(N^{\prime}\right)$, say $I^{\prime}$. Then, by [5, Theorem 4.3], $I^{\prime}$ corresponds to a sub-Hopf algebra of $H_{N^{\prime}}$, hence an intermediate field in $L / K$ which is realizable with respect to $H_{N^{\prime}}$. Conversely, if $F$ is a field which is realizable with respect to $H_{N^{\prime}}$, there is a corresponding o-stable subgroup of $\mathfrak{B}\left(N^{\prime}\right)$, hence of $\mathfrak{B}^{\prime}$, giving us a quasi-ideal of $\mathfrak{B}$.

By [11, Prop. 2.2] (which itself is a reformulation of the ideas from [7, §5]), sub-Hopf algebras of $H_{N}$ correspond bijectively to $G$-stable subgroups $I$ of $N$, hence realizable fields correspond to such $I$. We can relate this theory to [5] as follows. Suppose $\mathfrak{B}=(B, \cdot, \circ)$ is a brace, and $I$ is a o-stable subgroup of $\mathfrak{B}$. Let $G=(B, \circ)$, and let $N=\left\{\eta_{x}: x \in B\right\} \leq \operatorname{Perm}(G)$ where $\eta_{x}[y]=x \cdot y$. Let $I_{*}=\left\{\eta_{i}: i \in I\right\} \leq N$. Then

$$
{ }^{x} \eta_{i}=\eta_{(x \circ i) x^{-1}}, x, i \in B
$$

and since $I$ is o-stable we know $(x \circ i) x^{-1} \in I$, hence $I_{*}$ is $G$-stable. It is easy to see that the converse holds as well.

Additionally, if $I \unlhd N$ then $L^{H_{I}} / K$ is also Hopf-Galois for a particular Hopf algebra related to $H_{N}$-see [11, Theorem 2.10]. Thus we get:

Lemma 5.4. There is a bijection between -quasi-ideals $I$ of $\mathfrak{B}:=\mathfrak{B}(N)$ and intermediate fields $K \leq L_{I} \leq L$ realizable with respect to $H_{N^{\prime}}$ such that $L_{I} / K$ is also Hopf-Galois via the $K$-Hopf algebra $L_{I}\left[N^{\prime} / I^{\prime}\right]^{G}$, where $I^{\prime}$ is the image of $I$ under the isomorphism $I \mapsto I^{\prime}$ above.

How $L_{I}\left[N^{\prime} / I^{\prime}\right]^{G}$ acts on $L_{I}$ is not obvious-see the discussion prior to [11, Theorem 2.10] for a complete description.

Of course, if $I \unlhd(B, \circ)$, then the corresponding subgroup of $G$ is also normal. This gives:

Lemma 5.5. o-quasi-ideals $I$ of $\mathfrak{B}:=\mathfrak{B}(N)$ correspond bijectively with intermediate fields $K \leq$ $L_{I} \leq L$ realizable with respect to $H_{N^{\prime}}$ such that $L_{I} / K$ is Galois.

We summarize:

Theorem 5.6. Let $L / K$ be Galois, group $G$, and let $N \leq \operatorname{Perm}(G)$ be regular and $G$-stable. Let $\mathfrak{B}=\mathfrak{B}(N)$ and $\mathfrak{B}^{\prime}=(\mathfrak{B}(N))^{\prime}=\mathfrak{B}\left(N^{\prime}\right)$. Let $I \subseteq \mathfrak{B}$ be a quasi-ideal. Then there exists a field $K \leq L_{I} \leq L$ such that $L / L_{I}$ is Hopf-Galois via the $L_{I}$-Hopf algebra $L[I]^{G}$. Furthermore:

1. If $I$ is a -Q QI then $L_{I} / K$ is also Hopf-Galois with respect to a Hopf algebra which depends on $H$.

2. If $I$ is a $\circ-Q I$ then $L_{I} / K$ is (classically) Galois.

3. If $I$ is an ideal, then $L_{I} / K$ is both Galois and Hopf-Galois in the sense mentioned above.

Furthermore, any realizable intermediate field $F$ is of the form $L_{I}$ for some quasi-ideal $I$; and if $F$ satisfies the proprieties (1), (2), or (3) above, then I is a-QQI, o-QI, or an ideal respectively.

Example 5.7. Suppose $\mathfrak{B}=(B, \cdot, \cdot)$ is the trivial brace. If $I \leq(B, \cdot)$ is any subgroup, then $I$ is automatically a quasi-ideal since $x^{-1}(x \circ y)=y$. It is an ideal if and only if $I$ is normal in $(B, \cdot)$. This makes sense since $\mathfrak{B}^{\prime}$ is (isomorphic to) the brace corresponding to the classical Galois structure: each subgroup gives an intermediate field, and the Hopf-Galois structure on $L_{I}$ coincides with the Galois structure when $I$ is normal. 
Example 5.8. Suppose $\mathfrak{B}=\left(B,{ }^{\prime}, \cdot\right)$ is the almost trivial brace. If $I \leq(B, \cdot)$ is any subgroup, then $I$ is a quasi-ideal if and only if $x^{-1} .^{\prime}(x \circ y)=x y x^{-1}$ for all $x \in B, y \in I$, i.e., if $I$ is normal in $(B, \cdot)$. If this is the case, then it is automatically an ideal as well.

Example 5.9. Let $B=\langle\eta, \pi\rangle \cong D_{4}$ with, as usual,

$$
\eta^{i} \pi^{j} \circ \eta^{k} \pi^{\ell}=\eta^{k+(-1)^{\ell} i+2 j \ell} \pi^{j+\ell}, 0 \leq i, k \leq 3,0 \leq j, \ell \leq 1 .
$$

Of course, $I=\left\{1_{B}\right\}$ and $I=B$ are ideals. The group $(B, \cdot)$ has eight other subgroups. Notice that any quasi-ideal $I$ of $\mathfrak{B}$ of order 4 is necessarily an ideal of $\mathfrak{B}$ since $(B: I)=2$.

$I=\langle\eta\rangle$. We have $\left(x_{j}\right)^{-1}\left(x_{j} \circ \eta^{k}\right)=x_{j}^{-1} \eta^{k} x_{j} \in I$ since $I$ is normal in $(B, \cdot)$. Thus $I$ is a quasi-ideal, hence an ideal since $|I|=4$.

$I=\left\langle\eta^{2}\right\rangle$. This must be a quasi-ideal as well from the work above, as well as an ideal since $I=$ $Z(B, \cdot)=Z(B, \circ)$.

$I=\left\langle\eta^{k} \pi\right\rangle, 0 \leq k \leq 3$. Since

$$
(\eta \pi)^{-1}\left(\eta \pi \circ \eta^{k} \pi\right)=\eta \pi\left(\eta^{k-1}\right)=\eta^{2-k} \pi \notin\left\langle\eta^{k} \pi\right\rangle
$$

we see that $I$ is not a quasi-ideal.

$I=\left\langle\eta^{2}, \pi\right\rangle$. From the above, the quasi-ideal condition $x^{-1}(x \circ y) \in I$ holds for $y=\eta^{2}$. For $k=0,2$ we have

$$
\left(\eta^{i} \pi^{j}\right)^{-1}\left(\eta^{i} \pi^{j} \circ \eta^{k} \pi\right)=\pi^{-j} \eta^{-i}\left(\eta^{i+k} \pi^{j+1}\right)=\eta^{k} \pi \in I
$$

so $I$ is a quasi-ideal of $\mathfrak{B}$, hence an ideal.

$I=\left\langle\eta^{2}, \eta \pi\right\rangle$. For $k=1,3$ we have

$$
\left(\eta^{i} \pi^{j}\right)^{-1}\left(\eta^{i} \pi^{j} \circ \eta^{k} \pi\right)=\pi^{-j} \eta^{-i}\left(\eta^{i-k} \pi^{j+1}\right)=\eta^{(-1)^{j} k} \pi \in I
$$

and is also an ideal.

\section{Self-Opposite Braces}

We conclude this paper with a discussion concerning self-opposite braces. Of course, $\mathfrak{B}=\mathfrak{B}^{\prime}$ if and only if $(B, \cdot)$ is an abelian group. However, it is possible for $\mathfrak{B}$ and $\mathfrak{B}^{\prime}$ to be isomorphic, as the following example shows.

Example 6.1. Let $\left(G, *_{G}\right)$ be any nonabelian group. Let $B=G \times G$, and define two operations on $B$ as follows:

$$
\begin{aligned}
(x, y) \cdot(z, w) & =\left(x *_{G} z, w *_{G} y\right) \\
(x, y) \circ(z, w) & =\left(x *_{G} z, y *_{G} w\right) .
\end{aligned}
$$

It is easy to show that $\mathfrak{B}:=(B, \cdot, \circ)$ is a brace (indeed, the product of the trivial and almost trivial braces on $G)$, and that the map $T: \mathfrak{B} \rightarrow \mathfrak{B}^{\prime}$ given by $T(x, y)=(y, x)$ is an isomorphism. 
More generally, if $\mathfrak{B}$ is any brace, then so is $\mathfrak{B} \times \mathfrak{B}^{\prime}$, and $\left(\mathfrak{B} \times \mathfrak{B}^{\prime}\right)^{\prime}=\left(\mathfrak{B}^{\prime} \times \mathfrak{B}\right) \cong \mathfrak{B} \times \mathfrak{B}^{\prime}$. While equality, not isomorphism, is required for $R_{\mathfrak{B}}$ and $R_{\mathfrak{B}^{\prime}}$ to be equal, the enumeration of realizable fields depends only on the isomorphism class of $\mathfrak{B}$. Clearly, if $\mathfrak{B}(N)$ is self-opposite then quasi-ideal, etc. classify the realizable, etc., fields in the sense of Theorem 5.6 corresponding to the Hopf algebra $H_{N}$.

Because of this, it would be interesting to have sufficient, and possibly necessary, conditions for a brace to be self-opposite. While we do not have a full set of conditions (though certainly $(B, \cdot)$ abelian, or $\mathfrak{B} \cong \mathfrak{C} \times \mathfrak{C}^{\prime}$ suffice), we do have some necessary conditions, from which we can determine some braces which are not self-opposite.

For example, let us call $(x, y) \in B \times B$ an $L$-pair if $x \circ y=x y$; if $x \circ y=y x$ then we will call $(x, y)$ an $R$-pair. If $\phi: \mathfrak{B} \rightarrow \mathfrak{B}^{\prime}$ is an isomorphism and $(x, y)$ is an L-pair of $\mathfrak{B}$, then

$$
\phi(x) \circ \phi(y)=\phi(x \circ y)=\phi(x \cdot y)=\phi(x) \cdot \phi(y)=\phi(y) \cdot \phi(x)
$$

and hence $(\phi(x), \phi(y))$ is an $\mathrm{R}$-pair of $\mathfrak{B}$. Thus we get:

Proposition 6.2. If the number of L-pairs and R-pairs of $\mathfrak{B}$ is not equal, then $\mathfrak{B}$ is not selfopposite.

Example 6.3. Let us consider Example 2.4 one last time: $B=\langle\eta, \pi\rangle \cong D_{4}$ with

$$
\eta^{i} \pi^{j} \circ \eta^{k} \pi^{\ell}=\eta^{2 j \ell}\left(\eta^{k} \pi^{\ell}\right)\left(\eta^{i} \pi^{j}\right)=\eta^{k+(-1)^{\ell} i+2 j \ell} \pi^{j+\ell}, 0 \leq i, k \leq 3,0 \leq j, \ell \leq 1 .
$$

If $\eta^{i} \pi^{j} \circ \eta^{k} \pi^{\ell}=\eta^{i} \pi^{j} \eta^{k} \pi^{\ell}=\eta^{i+(-1)^{j} k} \pi^{j+\ell}$ then we must have

$$
k+(-1)^{\ell} i+2 j \ell \equiv i+(-1)^{j} k \quad(\bmod 4)
$$

Picking $j=\ell=0$ gives us 16 L-pairs. If $j=1, \ell=0$ we get

$$
k+i \equiv i-k \quad(\bmod 4)
$$

which provides 8 pairs, corresponding to the cases $k=0,2$. Setting $j=0, \ell=1$ gives another 8 pairs, and if $j=\ell=1$ we get

$$
k-i+2 \equiv i-k \quad(\bmod 4),
$$

so $2(i+k) \equiv 2(\bmod 4)$, which holds if $i$ and $k$ are of different parity, giving another 8 pairs. In total, $\mathfrak{B}$ has 40 L-pairs.

On the other hand, if $\eta^{i} \pi^{j} \circ \eta^{k} \pi^{\ell}=\eta^{2 j \ell}\left(\eta^{k} \pi^{\ell}\right)\left(\eta^{i} \pi^{j}\right)=\eta^{k} \pi^{\ell} \eta^{i} \pi^{j}, j, \ell=0,1$ then it is necessary and sufficient that $2 j \ell=0$, in other words either $j=0$ or $\ell=0$ or both. This gives $48 \mathrm{R}$-pairs for $\mathfrak{B}$, hence $\mathfrak{B}$ is not self-opposite.

\section{Acknowlegements}

Funding: The second author was supported in part by the London Mathematical Society [Grant \#41847].

The first author would like to thank Keele University for its hospitality during the development of this paper. 


\section{References}

[1] L. Guarnieri, L. Vendramin, Skew braces and the Yang-Baxter equation, Math. Comp. 86 (307) (2017) 2519-2534. doi:10.1090/mcom/3161. URL https://doi.org/10.1090/mcom/3161

[2] W. Rump, Braces, radical rings, and the quantum Yang-Baxter equation, J. Algebra 307 (1) (2007) 153-170. doi:10.1016/j.jalgebra.2006.03.040.

URL https://doi.org/10.1016/j.jalgebra.2006.03.040

[3] D. Bachiller, Counterexample to a conjecture about braces, J. Algebra 453 (2016) 160-176. doi:10.1016/j.jalgebra.2016.01.011.

URL https://doi.org/10.1016/j.jalgebra.2016.01.011

[4] A. Smoktunowicz, L. Vendramin, On skew braces (with an appendix by N. Byott and L. Vendramin), J. Comb. Algebra 2 (1) (2018) 47-86. doi:10.4171/JCA/2-1-3.

URL https://doi.org/10.4171/JCA/2-1-3

[5] L. N. Childs, Skew braces and the Galois correspondence for Hopf Galois structures, J. Algebra 511 (2018) 270-291. doi:10.1016/j.jalgebra.2018.06.023.

URL https://doi.org/10.1016/j.jalgebra.2018.06.023

[6] L. N. Childs, Taming wild extensions: Hopf algebras and local Galois module theory, Vol. 80 of Mathematical Surveys and Monographs, American Mathematical Society, Providence, RI, 2000 .

[7] C. Greither, B. Pareigis, Hopf Galois theory for separable field extensions, J. Algebra 106 (1) (1987) 239-258. doi:10.1016/0021-8693(87)90029-9.

URL http://dx.doi.org/10.1016/0021-8693(87)90029-9

[8] P. J. Truman, Canonical nonclassical Hopf-Galois module structure of nonabelian Galois extensions, Comm. Algebra 44 (3) (2016) 1119-1130.

URL https://doi.org/10.1080/00927872.2014.999930

[9] S. Taylor, P. J. Truman, The structure of Hopf algebras giving Hopf-Galois structures on quaternionic extensions, New York J. Math. 25 (2019) 219-237.

[10] P. J. Truman, Commuting Hopf-Galois structures on a separable extension, Comm. Algebra 46 (4) (2018) 1420-1427. doi:10.1080/00927872.2017.1346107.

URL https://doi.org/10.1080/00927872.2017.1346107

[11] A. Koch, T. Kohl, P. J. Truman, R. Underwood, Normality and short exact sequences of HopfGalois structures, Comm. Algebra 47 (5) (2019) 2086-2101. doi:10.1080/00927872.2018. 1529237.

URL https://doi.org/10.1080/00927872.2018.1529237 